\title{
Conjugative type IVb pilus recognizes lipopolysaccharide of recipient cells to initiate PAPI-1 pathogenicity island transfer in Pseudomonas aeruginosa
}

Toan Phuoc Hong ${ }^{1}$, Michelle Q. Carter ${ }^{2}$, Paolo Struffi ${ }^{1}$, Stefano Casonato ${ }^{1}$, Youai Hao ${ }^{3}$, Joseph S. Lam³, Stephen Lory ${ }^{2}$ and Olivier Jousson ${ }^{1 *}$ (iD

\begin{abstract}
Background: Pseudomonas aeruginosa pathogenicity island 1 (PAPI-1) is one of the largest genomic islands of this important opportunistic human pathogen. Previous studies have shown that PAPI-1 encodes several putative virulence factors, including a major regulator of biofilm formation and antibiotic-resistance traits. PAPI-1 is horizontally transferable into recipient strains lacking this island via conjugation mediated by the specialized type IV pilus. The PAPI-1 encodes a cluster of ten genes associated with the synthesis and assembly of the type IV pilus. The PAPI-1 acquisition mechanism is currently not well understood.

Results: In this study, we performed a series of conjugation experiments and identified determinants of PAPI-1 acquisition by analyzing transfer efficiency between the donor and a series of mutant recipient strains. Our data show that common polysaccharide antigen (CPA) lipopolysaccharide (LPS), a homopolymer of D-rhamnose, is required for initiating PAPI-1 transfer, suggesting that this structure acts as a receptor for conjugative type IV pilus in recipient strains. These results were substantiated by experimental evidence from PAPI-1 transfer assay experiments, in which outer membrane or LPS preparations from well-defined LPS mutants were added to the transfer mix to assess the role of $P$. aeruginosa LPS in PAPI-1 transfer and in vitro binding experiments between pilin fusion protein GST-pilV2' and immobilized LPS molecules were performed. Our data also showed that $P$. aeruginosa strains that had already acquired a copy of PAPI- 1 were unable to import additional copies of the island, and that such strains produced proportionally lower amounts of CPA LPS compared to the strains lacking PAPI-1.
\end{abstract}

Conclusions: These results suggest that a PAPI-1 exclusion mechanism exists in $P$. aeruginosa that might serve to regulate the avoidance of uncontrolled expansions of the bacterial genome.

Keywords: Pseudomonas aeruginosa, PAPI-1 pathogenicity island, Horizontal gene transfer

\section{Background}

Horizontal gene transfer (HGT) mediated by microorganisms is a major evolutionary mechanism for the acquisition of new functionalities. HGT allows rapid and drastic changes in bacterial genomes, as up to hundreds of new genes can be acquired during a single genetic exchange event. HGT is recognized to play an important role in the evolution of virulence, antibiotic resistance

\footnotetext{
* Correspondence: olivier.jousson@unitn.it

${ }^{1}$ Centre for Integrative Biology, University of Trento, 38123 Trento, Italy

Full list of author information is available at the end of the article
}

and adaptation to the new environments $[1,2]$. The acquisition of virulence genes may radically alter the disease-causing potential of a microorganism. In some instances, acquisition of a single gene or a small cluster of genes encoding critical virulence determinants may be the only genetic difference between avirulent and virulent strains of the same species [3, 4]. Virulence genes are often part of a large block of DNA referred to as genomic islands (GIs). GIs are accessory genomic segments present only in certain bacterial strains; they are often flanked by direct repeats and inserted in the vicinity of tRNA genes. 
Reversible excision and integration further implicate their potential for inter-bacterial transfer [5]. Those GIs that lead to enhancement of fitness in a host organism are called pathogenicity islands [6]. Integrative and conjugative elements (ICEs), or conjugative transposons, are well-characterized GIs that in many cases have retained mobility [7, 8]. In contrast, a number of GIs appear to belong to the group of ancient ICEs that became fixed in the bacterial chromosome due to degeneration of their conjugative elements by deletion of integration sites or mutations in genes encoding transfer functions [9]. The best-characterized ICEs to date contain specific features associated with conjugative plasmids and bacteriophages; can be transferred horizontally following recognition of the recipient cell by the donor utilizing a conjugative mechanism that, in many instances, is associated with the type IV protein secretion pathway [10]. The recipient cell is recognized by the pilus structure that is part of the type IV secretion apparatus of the donor [11].

Pseudomonas aeruginosa is highly adaptable to survive in a wide range of environmental niches; this ability is reflected by its large genomic repertoire. Indeed, the genome database for $P$. aeruginosa strains available to date show that this species possesses a large core genome of ca. 5000 conserved genes and an accessory gene pool of 1000-1500 additional genes; most of the latter are known to be arranged in a limited number of genomic islands [12]. PAPI-1 is one of the largest GIs characterized in $P$. aeruginosa PA14 [13], a highly virulent strain that can infect a broad range of plants, insects, and animals. It is integrated at the $a t t B$ site, juxtaposed to tRNA-lys genes [14] and consisted of a cluster of 108 genes that encode a number of virulence determinants, whose disruption resulted in the attenuation of the virulence phenotype in several infection models [13]. In addition, PAPI-1 carries several regulatory genes, such as the one that encodes PvrSR/RcsCB two-component system, which controls biofilm formation and dispersal in $P$. aeruginosa strains causing chronic infections in individuals with cystic fibrosis [15]. PAPI-1 island is present in wild-type PA14 strain, whereas, it is not found in PAO1. However, it can easily be transferred from PA14 to PAO1. PAPI-1 transfer has previously been described as a conjugation process mediated by type IVb pilus in co-culture experiments with donor and recipient cells $[14,15]$. Type IVb pilus is encoded by a 10-gene cluster in PAPI-1 [15] and is closely related to the genes found in the enterobacterial plasmid R64. Previous studies on conjugal plasmid R64 suggested that the thin pilus, PilV adhesin, was formed by a recombinant mechanism between various cassettes, and a shufflon [16] presumably recognizes a specific structure of the lipopolysaccharide molecules of recipient cells, determining the transfer specificity of the plasmid R64 [17].
The aim of this study was to investigate the mechanism of acquisition of PAPI-1 in P. aeruginosa. We performed a series of conjugation experiments on wild-type or mutant donor and recipient strains, and analyzed the determinants that dictate PAPI- 1 transfer efficiency. We demonstrated for the first time that the conjugative type IVb pilus of the donor can recognize CPA LPS on the recipient outer membrane, and that this structure is required to initiate the transfer of PAPI-1. Our data also indicate that $P$. aeruginosa strains containing PAPI-1 specify a mechanism to exclude additional copies of PAPI-1 by shutting down the CPA LPS biosynthesis.

\section{Methods}

\section{Strains, plasmids, and culture conditions}

All strains and plasmids used in this study are listed in Additional file 1: Table S1 and Additional file 2: Table S4. $P$. aeruginosa strains and mutants were grown in lysogeny broth (LB, also called Luria-Bertani medium) (Sigma-Aldrich) supplemented with appropriate antibiotics. For selection of $P$. aeruginosa mutants, the antibiotics used were gentamicin and tetracycline, both at a concentration of $75 \mu \mathrm{g} / \mathrm{ml}$. For maintenance of plasmids in Escherichia coli, the medium was supplemented with ampicillin at $100 \mu \mathrm{g} / \mathrm{ml}$ and chloramphenicol at $34 \mu \mathrm{g} / \mathrm{ml}$. Isopropyl-D-thiopyranoside (IPTG) was added at a final concentration of $0.5 \mathrm{mM}$ to induce GST-pilV2' expression in the pGEX-2 T plasmid.

\section{Construction of PA14ATnC2:: $\mathrm{Tc}^{\mathrm{R}}$ mutant}

The mutant PA14 $\triangle T n C 2:: \mathrm{Gm}^{\mathrm{R}}$, derived from PA14 transposon insertion mutant library [18] in which the transposon was inserted at nucleotide 1634 of the PAPI1 gene RL090 (PA14_59200), is proficient in transfer of the PAPI-1 and has been used as a donor in PAPI-1 transfer assay [15]. In some experiments, a donor with tetracycline-resistant $\left(\mathrm{Tc}^{\mathrm{R}}\right)$ marker was required since the recipient contained a gentamicin-resistance $\left(\mathrm{Gm}^{\mathrm{R}}\right)$ gene, and one would expect to have an equivalent transfer efficiency to the PA14 $\triangle \operatorname{Tn} C 2:: \mathrm{Gm}^{\mathrm{R}}$. The knockout mutant was constructed by using gene replacement vectors as previously described [19]. All primers used for generating the mutant PA14 $\triangle T n C 2:: \mathrm{Tc}^{\mathrm{R}}$ are listed in Additional file 2: Table S4. Briefly, a cassette conferring $\mathrm{Tc}^{\mathrm{R}}$ flanked by two DNA fragments of about $500 \mathrm{bp}$ flanking the PA14_59200 gene was cloned in the vector pJET1.2 before subcloning into the vector pEXG2. The recombinant plasmid was conjugated from $E$. coli $\lambda$ pir S17.1 into P. aeruginosa [20]. The integrative plasmids were selected on LB agar medium supplemented with gentamicin, tetracycline or irgasan at $25 \mu \mathrm{g} / \mathrm{ml}$. To resolve merodiploids, a second selection round on LB agar medium with $6 \%$ sucrose was performed. 
Transformants were screened by colony PCR and confirmed by DNA sequencing.

\section{Screening for PAO1 mutants deficient in PAPI-1 acquisition}

A standard PAPI-1 transfer assay via liquid mating was carried out as previously described [15]. Briefly, mutant PA14 $\triangle T n C 2:: \mathrm{Gm}^{\mathrm{R}}$ was used as donor and a series of PAO1 mutants, with altered LPS biosynthesis, obtained from a PAO1 transposon mutant library were used as recipients (Additional file 3: Table S2). After overnight growth at $37^{\circ} \mathrm{C}$ with shaking at $200 \mathrm{rpm}$, the donor cells were harvested at an $\mathrm{OD}_{600}$ of 0.8 and were mixed with the recipient cells at an $\mathrm{OD}_{600}$ of 0.4 , spun down and resuspended in $1 \mathrm{ml}$ of fresh LB without antibiotics. The mating mixture was incubated in $15-\mathrm{ml}$ culture tubes, statically at $37{ }^{\circ} \mathrm{C}$ for $24 \mathrm{~h}$. The mating mixture was diluted to appropriate dilutions and plated on LB agar medium supplemented with gentamicin and tetracycline at $75 \mu \mathrm{g} / \mathrm{ml}$ to select for transconjugants, and on LB agar plates containing tetracycline at $75 \mu \mathrm{g} / \mathrm{ml}$ to select for recipients. The transfer efficiency was calculated as a ratio of transconjugants to recipients colonies in the mating mixture.

\section{Outer membrane (OM) preparation}

The outer membrane of $P$. aeruginosa was isolated by using sodium lauroylsarkosinate (sarkosyl) as previously described [21]. Briefly, cultures of $P$. aeruginosa were grown overnight at $37{ }^{\circ} \mathrm{C}$ with shaking at $200 \mathrm{rpm}$ in LB broth. The pre-inoculum was then diluted 100-fold in fresh LB medium and grown at $37{ }^{\circ} \mathrm{C}$ with shaking at $200 \mathrm{rpm}$ to an $\mathrm{OD}_{600}$ of 1.0. Cells were harvested and resuspended in $15 \mathrm{ml}$ lysis buffer containing $20 \mathrm{mM}$ Tris-Cl pH 7.5, $100 \mathrm{mM} \mathrm{NaCl}, 1 \mathrm{mM}$ EDTA, lysozyme $0.5 \mathrm{mg} / \mathrm{ml}$ and a complete protease inhibitor cocktail (Roche). The lysate was sonicated and centrifuged at $10,000 \times g, 10 \mathrm{~min}, 4{ }^{\circ} \mathrm{C}$ to remove cellular debris. The membrane fraction was isolated by ultracentrifugation $\left(200,000 \times g\right.$ at $4{ }^{\circ} \mathrm{C}$ for $\left.60 \mathrm{~min}\right)$. The pellets containing inner and outer membranes were further fractionated at $100,000 \times g$ for $30 \mathrm{~min}$ after incubation with $0.2 \%$ sarkosyl. The pellet contained outer membranes, which were finally resuspended in Tris-Cl buffer $20 \mathrm{mM} \mathrm{pH} 7.5$ and resolved on $12 \%$ sodium dodecyl sulfate (SDS)-polyacrylamide gels and stained with Coomassie blue. The concentration of OM was measured by the standard Lowry method using $D C^{\mathrm{TN}}$ Protein Assay kit (BIO-RAD) with BSA protein as a standard.

\section{LPS preparation}

For pilin-LPS binding assays, LPS from various $P$. aeruginosa strains and mutants were prepared by using the hot phenol-water extraction protocol from Westphal and Jann (1965) with minor modifications [22]. Briefly, cell suspensions in $100 \mathrm{mM} \mathrm{NaCl}$ were first heated to $68{ }^{\circ} \mathrm{C}$ before adding an equal volume of hot phenol and stirring vigorously for $2 \mathrm{~h}$ at $68{ }^{\circ} \mathrm{C}$. LPS was then fractionated by centrifugation at $12,000 \times g$ for $15 \mathrm{~min}$, at $4{ }^{\circ} \mathrm{C}$. LPS in the upper phase was recovered and dialyzed against water to remove residual phenol. LPS extract was further treated with DNase, RNase and proteinase $\mathrm{K}$ to eliminate contaminations. LPS extract was finally lyophilized before use. The LPS samples were analyzed by SDS-PAGE and silver staining [23]. Quantitation of LPS was performed by using a Kdo assay as described previously [24].

\section{PAPI-1 transfer inhibition assay}

$\mathrm{OM}$ and LPS preparations at various concentrations $(0 ; 0.5 ; 1.0 ; 2.0 ; 5.0 ; 10.0$ and $15.0 \mu \mathrm{g})$ were added to a standard mating assay based on plasmid conjugal transfer [25], between the donor PA14 $\triangle T n C 2:: \mathrm{Gm}^{\mathrm{R}}$ and the recipient PAO1:: $\mathrm{Tc}^{\mathrm{R}}$. A mating mixture without the addition of OM or LPS was also included as a negative control for this experiment. The transfer inhibition index was calculated by dividing the transfer efficiency observed with the addition of OM or LPS to that of the control.

\section{Western blotting for LPS samples}

LPS samples prepared by the Hitchcock and Brown method [26], were used for SDS-PAGE, silver staining and Western immunoblotting, following protocols that had been described previously [22]. Briefly, $3 \mu \mathrm{l}$ of LPS samples was loaded into the 12\% SDS-PAGE and run at $80 \mathrm{~V}$ for $120 \mathrm{~min}$. LPS was electrophoretically transferred onto nitrocellulose membrane at $180 \mathrm{~mA}$ for $50 \mathrm{~min}$. The membrane was then blocked with $5 \%$ skim milk for $30 \mathrm{~min}$ at room temperature. The membranes were washed in PBS for $3 \times 10 \mathrm{~min}$; the primary antibodies monoclonal antibodies (MAb) against different regions of the LPS structure, including MF15-4 (O-specific antigen specific), N1F10 (CPA specific), 5c101 (outer core specific), and 5c-7-4 (inner core specific), were added and incubated overnight at room temperature. Following a $3 \times 10 \mathrm{~min}$ PBS wash, secondary antibodies were added for an additional hour. The membrane was washed for $2 \times 10 \mathrm{~min}$ in PBS, $10 \mathrm{~min}$ in buffer A before developed by BCIP/NBT detection kit.

\section{Expression and purification of GST-pilV2' fusion protein}

The C-terminal region of pilV2 gene encoding 97 residues was amplified with the primers listed in Additional file 2: Table S4 and cloned into PJET 1.2/ Blunt. The insert was then subcloned into the expression vector pGEX-2 $\mathrm{T}$ (Life Technologies) and transformed into E. coli BL21. E. coli BL21 containing pGEX-2 T-pilV2' 
was grown to an $\mathrm{OD}_{600}$ of 0.6 at $37{ }^{\circ} \mathrm{C}$ with shaking at $200 \mathrm{rpm}$, before adding IPTG to induce expression of GST-pilV2' protein. The culture was incubated for additional $3 \mathrm{~h}$. The cells were collected by centrifugation at $12,000 \times g$, at $4{ }^{\circ} \mathrm{C}$ for $20 \mathrm{~min}$. The GST-pilV2' fusion protein was then purified by using glutathione Sepharose 4B (GE Healthcare), as previously described [27].

\section{Microtiter plate binding assay}

Binding of LPS to GST-pilV2' fusion was quantified by a modified enzyme-linked immunosorbent assay (ELISA) essentially as described previously [28]. Microtiter plates (Corning) were coated with $10 \mu \mathrm{g} / \mathrm{ml}$ LPS from PAO1 and PAO1 with PAPI-1 suspended in PBS $(0.137 \mathrm{M} \mathrm{NaCl}$, $0.005 \mathrm{M} \mathrm{KCl}, 0.009 \mathrm{M} \mathrm{Na}_{2} \mathrm{HPO}_{4}$, and $0.001 \mathrm{M} \mathrm{KH}_{2} \mathrm{PO}_{4}$ (pH 7.4)) containing 0.05\% Tween 20 (v/v, PBST). The plates were then washed with PBST and blocked with $3 \%$ BSA. GST-pilV2' fusion was added to the wells coated with LPS and incubated for $2 \mathrm{~h}$ at room temperature. After three washes with PBST, mouse anti-GST antibody was added and incubated for $1.5 \mathrm{~h}$ following three washes with PBST, HRP-labeled anti-mouse Ig (Sigma-Aldrich) was added for $1 \mathrm{~h}$, followed by three additional washes. A solution of 3,3',5,5'-tetramethylbenzidine (Thermo Scientific) was used for color development at $\mathrm{A}_{450}$. LPS from Salmonella enterica was used as a negative control.

\section{Results}

PAO1 derivatives defective in CPA LPS biosynthesis are deficient in the acquisition of PAPI-1

It has been known that PAPI-1 island is transferable from one to another P.aeruginosa strain lacking it by conjugation mechanism via type IVb pilus. PAPI-1 island carries a 10-gene cluster (pilL2, -N2, -O2, -P2, -Q2, $-R 2,-S 2,-T 2,-V 2$, and $-M 2)$ encoding structural and assembly accessory proteins of type IVb pilus such as PilS2, the major subunit of the pilus filament and PilV2, the minor subunit of the pilus filament. Insertion of the transposon into any of 10 pil genes led to a significant loss of PAPI-1 transfer efficiency [15]. Here, we confirmed that the transfer efficiency values of PA14 $\Delta$ pilV2 and PA14 $\Delta$ pilS2 are $<10^{-7}$, which is significantly lower than the positive control mutant PA14 $\triangle T n C 2:: \mathrm{Gm}^{\mathrm{R}}$, previously been proposed as a proficient donor in PAPI-1 transfer [15].

Since in conjugal plasmid system the donor pilus is known to interact with specific components of LPS on the recipient membrane to initiate the transfer [29], we examined the effects of using various PAO1 mutants for LPS biosynthesis as recipients on PAPI-1 transfer assay (Fig. 1). We first checked the transfer efficiency of PAPI-1 from PA14 $\triangle T n C 2:: \mathrm{Gm}^{\mathrm{R}}$ into the mutant $\mathrm{PAO} 1 \triangle \operatorname{algC}$, in which $\operatorname{algC}$ gene was disrupted by transposon insertion at nucleotide 628 [30]. The gene $\operatorname{algC}$ encodes a phosphoglucomutase, which is required for the synthesis of a complete LPS structure [31]. The mutant PAO1 $\triangle a \lg C$ thus produces a truncated LPS core and is devoid of $\mathrm{O}$-antigen. When the transfer assay was carried out between the donor PA14 $\triangle T n C 2:: \mathrm{Gm}^{\mathrm{R}}$ and PAO1 $\triangle$ algC:: $\mathrm{TC}^{\mathrm{R}}$ (PA5322), the transfer efficiency was reduced by three orders of magnitude compared to that of wild-type PAO1 (Fig. 1). This suggests that a complete LPS structure plays an important role in PAPI-1 acquisition. We therefore decided to screen a panel of $32 \mathrm{PAO} 1$

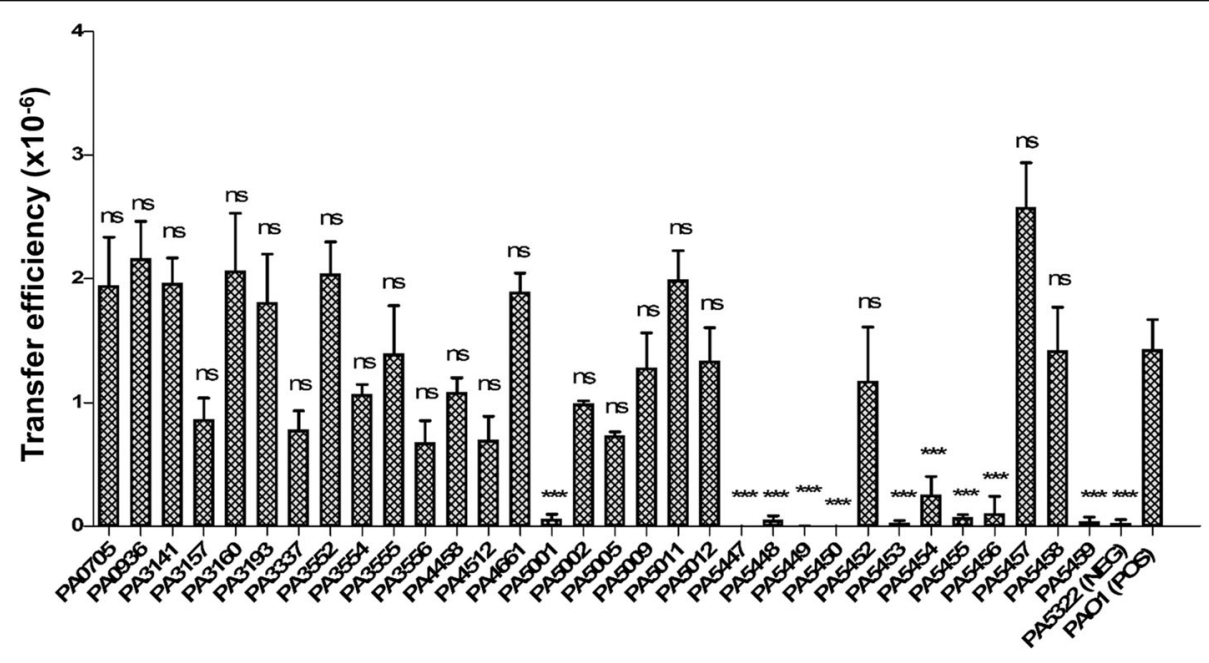

PA01 mutants for LPS biosynthesis

Fig. 1 PAPI-1 transfer efficiency into various PAO1 mutants for LPS biosynthesis as recipient strains. Positive control (POS): PAO1::TC ${ }^{R}$ and Negative control (NEG): PA5322 (or PAO1 $\triangle$ algC). Results were shown as mean \pm SD for three independent replicates. Statistical significance was assessed by One-way ANOVA compared to the positive control (ns: no significant difference; and ${ }^{* *} p<0.001$ ) 
mutants for LPS biosynthesis (Additional file 3: Table S2) [32] using them as recipients in the PAPI-1 transfer assay. These mutants were obtained from the PAO1 transposon mutant library, which contain $\mathrm{Tc}^{\mathrm{R}}$ [30].

The results showed that transfer efficiency of PAPI-1 into 10 PAO1 mutants including PA5001; PA5447 (wbpZ); PA5448 ( $w b p Y)$ ); PA5449 (wbpX); PA5450 (wzt); PA5453 (gmd); PA5454 (rmd); PA5455; PA5456 and PA5459 was significantly decreased by two to three orders of magnitude, compared to the PAO1 control. All these genes, except PA5001, were predicted as members of two operons [33]. The first one is at coordinates 6135968-6144991 (8 genes) and the other one at 6145399-6151151 (5 genes). Interestingly, these genes are known to be involved in Common Polysaccharide Antigen (CPA) biosynthesis (Additional file 4: Table S3). The genes algC, $w b p Z, w b p Y, w b p X, w z t, w b p M, g m d$, rmd encode enzymes involved in CPA biosynthesis [32], as shown in Additional file 5: Figure S1, while the genes PA5455, PA5456, PA5459 encode transferase enzymes [34]. The CPA or A-band LPS has been shown to contain a tri-saccharide repeating unit: $[\rightarrow 3)-\alpha-D-R h a-$ $(1 \rightarrow 3)-\alpha-\quad$ D-Rha- $(1 \rightarrow 2)-\alpha-\quad$ D-Rha- $(1 \rightarrow]_{n}$, so-called D-rhamnose homopolymer or rhamnan structure. This structure has been characterized by different authors with good agreement between different studies [35, 36]. Our data support the idea that the CPA could act as a receptor for conjugative type IV pilus as an initial step of PAPI-1 transfer. Noteworthy, the rmd mutant showed a slightly higher efficiency compared to the negative control which can be explained by the complementary effect of gmd gene. The RMD enzyme encoded by rmd gene catalyzes the final step in the GDP-D-Rha biosynthesis pathway. The disruption of rmd gene in $P$. aeruginosa chromosome impairs CPA synthesis [37], while gmd is partially capable of catalyzing the same reaction as RMD enzyme in vitro [38]. Therefore, gmd could complement CPA biosynthesis in the rmd mutant. We also observed that the transfer efficiency of the $w b p W$ mutant (PA5452) was comparable to the positive control. This is not surprising since it has been recently found that $P s l B$ gene is able to substitute $w b p W$ to promote CPA production in a $w b p W$ mutant [39] at a low abundance [37]. Here, to confirm if $p s l B$ plays a role in PAPI-1 transfer, we carried out transfer assay between PA14 $\triangle \mathrm{TnC} 2$ donor and those PAO1 mutants. Our result showed that the transfer efficiency of $\mathrm{PAO} 1 \Delta p s l B$ is comparable to $\mathrm{PAO} 1 \Delta w b p W$, while it is significantly decreased by about 20-fold as compared to that of PAO1 $\Delta w b p W / p s l B$ (Additional file 6: Figure S2 and Additional file 7: Table S5). The genes PA5455, PA5456, PA5459 are located in a five-gene operon (PA5455PA5459). The enzymes pa5455 and pa5456 were recently found to be essential for CPA biosynthesis [34]. Mutants in these genes are devoid of CPA, and show a reduced PAPI-1 transfer efficiency (Fig. 1). To the contrary, enzymes pa5457 and pa5458 are not essential for biosynthesis of a CPA structure [34], and mutants in these genes showed comparable transfer efficiency as the wild-type strain. Noticeably, the mutant PA5459 exhibited significantly reduced PAPI-1 transfer efficiency even though it is known to produce observable changes in CPA phenotypes. Mutants involved in core LPS synthesis such as waaC, waaF and waaP, did not show a significant decrease in PAPI-1 transfer. These results suggest that the CPA is the main LPS structure driving the contact and interaction between donor and recipient in $P$. aeruginosa.

\section{Addition of OM and CPA LPS preparations inhibits PAPI-1 transfer}

We postulated that either OM fractions or CPA LPS, the putative receptor for conjugative pilus, could compete with recipient cells for binding to the conjugative pilus; hence the use of these $P$. aeruginosa cell envelope components might block the transfer of PAPI-1 to the recipient. We prepared OM and LPS from isogenic mutants of $P$. aeruginosa PAO1; two of the mutants were defective in CPA LPS biosynthesis ( $\Delta r m d$ and $\triangle a \lg C$ ) and two other mutants were defective in OSA LPS ( $\triangle w b p M$ and $\Delta w z x)$. These preparations were added to the standard mixture of PAPI-1 transfer assay at different amount from 0 to $15 \mu \mathrm{g}$. OM and LPS preparations from OSA-mutants $(\Delta w b p M$ and $\Delta w z x)$ strongly inhibited transfer even at low concentrations $(<5 \mu \mathrm{g})$ and reached a maximum inhibition of about $80 \%$ (Fig. 2; Additional file 8: Table S7 and Additional file 9: Table S8). In contrast, OM preparation and LPS from CPA-mutants ( $\triangle r m d$ and $\triangle a l g C$ ) did not significantly affect the efficiency of PAPI-1 transfer. These results strongly support our hypothesis that CPA LPS in P. aeruginosa acts as a specific receptor for the IVb pilus, and is required to initiate the transfer of PAPI-1.

Recipient strains carrying PAPI-1 show a significant reduction in its ability to acquire additional copies of the island To address the question of whether $P$. aeruginosa strains already carrying PAPI-1 could acquire additional copies of this island, we carried out transfer assays using the donor PA14 $\triangle T n C 2:: \mathrm{Gm}^{\mathrm{R}}$ or PAO1Bla6TnC2:: $\mathrm{Gm}^{\mathrm{R}}$ and the recipients with PAPI-1 (PA14ATnC2::Tc ${ }^{\mathrm{R}}$ or PAO1Bla6TnC2::Tc ${ }^{\mathrm{R}}$ ) or without PAPI-1 (PA14 $\Delta$ soj and PAO1). The transconjugants carrying more than one copy of PAPI-1 were selected with double antibioticresistant markers (Gm and $\mathrm{Tc}$ ). Herein, we designated $[+]$ and $[-]$ for strains with and without PAPI-1, respectively. The transfer efficiency of PA14+ to PA14- is significantly lower than to that of the control PAO1(Fig. 3; Additional file 10: Table S6). This observation suggested that the transfer efficiency of PAPI-1 is strain- 


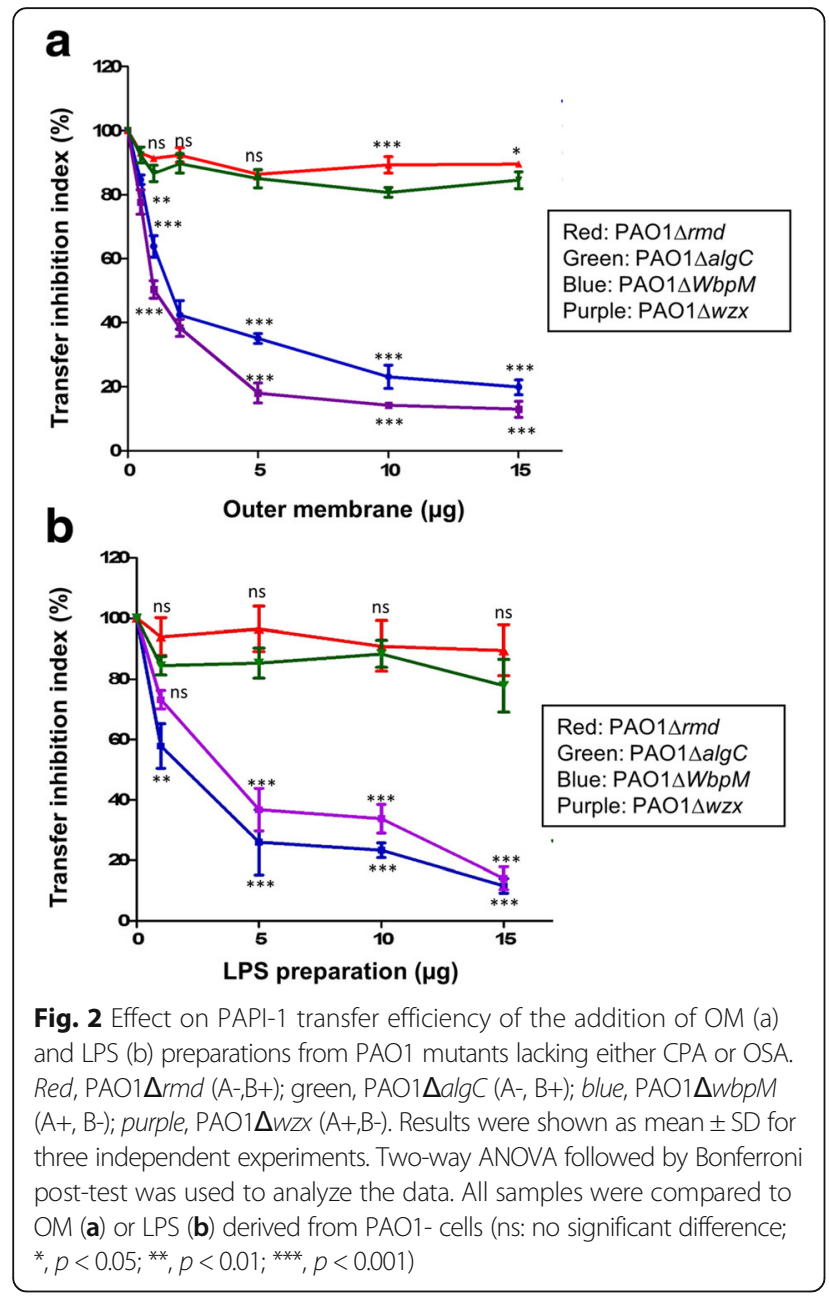

dependent. Interestingly, our data show that PAO1 strain carrying PAPI-1 can act as a donor of the island, transferring it to the control recipient (PAO1-) at an efficiency level comparable to the PA14+ donor. These results also demonstrated the ability of the recipient strains to acquire more than one copy of PAPI-1, even if this occurs at a much lower efficiency as compared to the control, with a decrease of one and three orders of magnitude for PA14+ to PA14+ and PAO1+ to PAO1+ transfers, respectively. Our data thus indicate that $P$. aeruginosa strains which already acquired PAPI-1 showed a significant decrease in their ability to receive additional copies of the island. This implies that $P$. aeruginosa strains carrying PAPI-1 specify a mechanism to exclude the acquisition of additional copies of the island.

The acquisition of PAPI-1 activates a surface exclusion mechanism

We hypothesized that after acquisition of PAPI-1, recipient $P$. aeruginosa strains would modify their surface to avoid further contact and subsequent transfer from the donor cells. To verify if the presence of

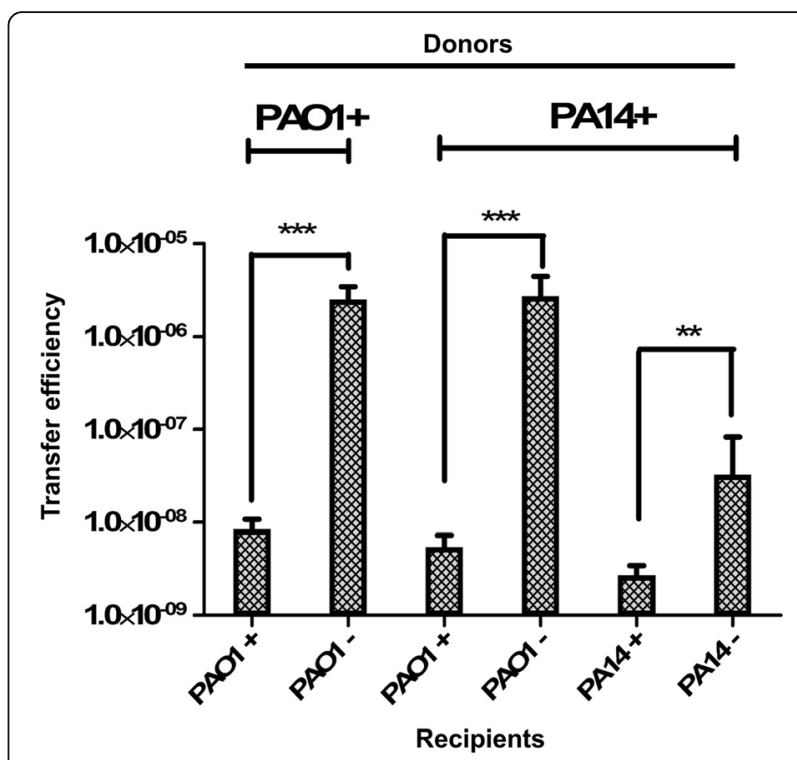

[+]: With PAPI-1

[-]: Without PAPI-1

Fig. 3 Transfer efficiency of multiple PAPI-1 copies into recipient strains. Marks [+] or [-] stand for strains with or without PAPI-1, respectively. After acquisition of PAPI-1, PAO1 becomes a stable donor which can transfer PAPI-1 to another recipient and decreases its ability of receiving additional copies of PAPI-1. Results are presented as mean \pm SD for three independent experiments. Statistical significance was calculated by the unpaired t-test ${ }^{* *} p<0.01$, and $\left.{ }^{* * *} p<0.001\right)$

PAPI-1 in the cell genome can affect the structure of its OM and LPS, we performed the standard transfer assay with the addition of OM and LPS, prepared from strains with and without PAPI-1. The addition of OM preparations derived from strains with PAPI-1 did not impact the transfer efficiency, when compared to the OM prepared from strains without the pathogenicity island (Fig. 4a). The effect of LPS preparations (Fig. 4b) is similar as that of OMs for strain PAO1, but not for PA14. Indeed, the addition of LPS from PA14- did not induce any significant variation in transfer efficiency. Experimental data were also shown in Additional file 8: Table S7 and Additional file 9: Table S8.

\section{Reduction of CPA LPS production plays a role in the surface exclusion mechanism of PAPI-1}

To better understand the putative mechanism utilized by $P$. aeruginosa to exclude additional copies of PAPI-1, we first considered potential alterations in surface exclusion. We therefore asked whether the strains containing PAPI-1 exhibits altered LPS structure, which would result in the reduction of the donor's pilus ability to bind to its receptor. Results from silver-stained SDS-PAGE of LPS did not show any discernible differences between 


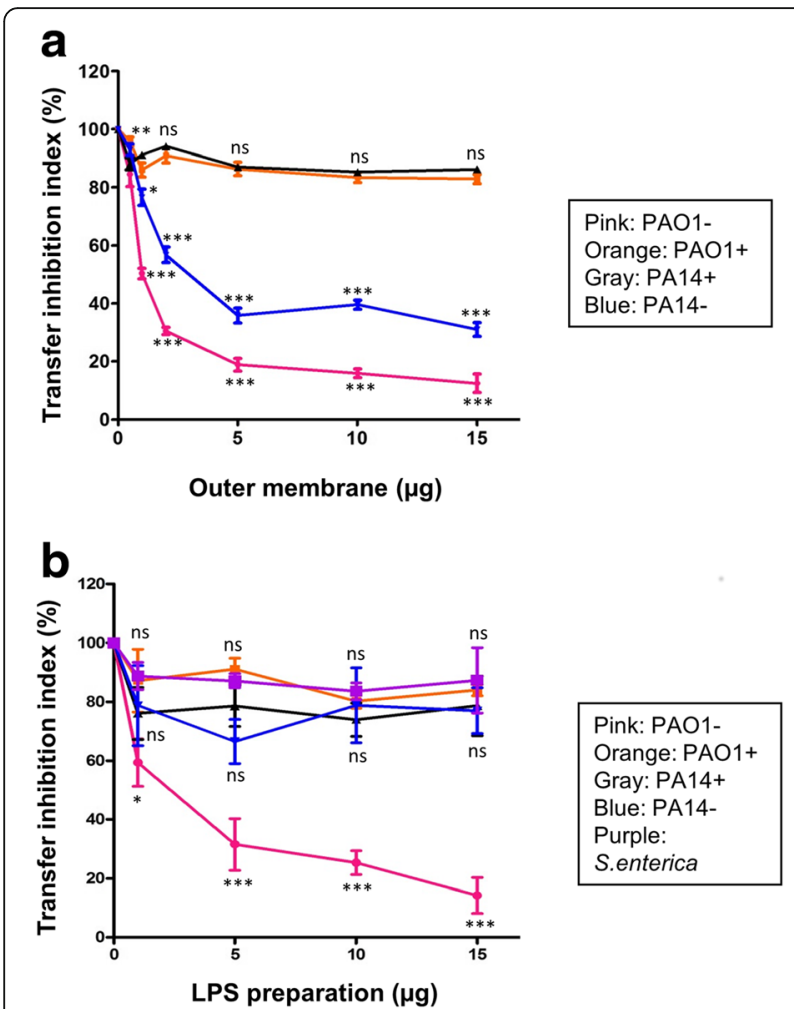

Fig. 4 Effect on PAPI-1 transfer of the addition of OM (a) and LPS (b) preparations derived from strains with (+) or without (-) PAPI-1 island. Pink, PAO1-; orange, PAO1+; black, PA14+; blue, PA14-. Purple data point on (b) are LPS from Salmonella enterica used as negative control. Results were shown as mean \pm SD for three independent experiments. Two-way ANOVA followed by Bonferroni post-test was used to analyze the data. All samples were compared to OM (a) or LPS (b) derived from PAO1- cells (ns: no significant difference; *, $P<0.05$; ${ }^{* *}, P<0.01$; ${ }^{* *}, P<0.001$ )

the LPS banding patterns in a given strain with or without PAPI-1 (Fig. 5a). We then performed Western immunoblotting using a combination of monoclonal antibodies (MAb) that recognized distinct regions within $P$. aeruginosa LPSs, including MF15-4 (serotype O5 OSA-specific) [40, 41]; N1F10 (CPA-specific) [40, 42]; 5c-7-4 (inner core-specific) [40, 43]; 5c-101 (outer corespecific) $[40,43]$. This could not be done for PA14 strains because the corresponding set of MAb are not available. We observed that there are no differences between different parts of LPS, with the notable exception of the CPA component from PAO1+ cells, which is significantly reduced compared to PAO1- cells (Fig. 5b). This result could be explained assuming that, after acquiring a PAPI-1 island, PAO1 downregulates the expression of genes involved in the biosynthesis of CPA LPS, leading to a reduced ability to bind the conjugative pilus. To test whether the PAO1+ strain had lost the ability to recognize the donor conjugative pilus, we over-

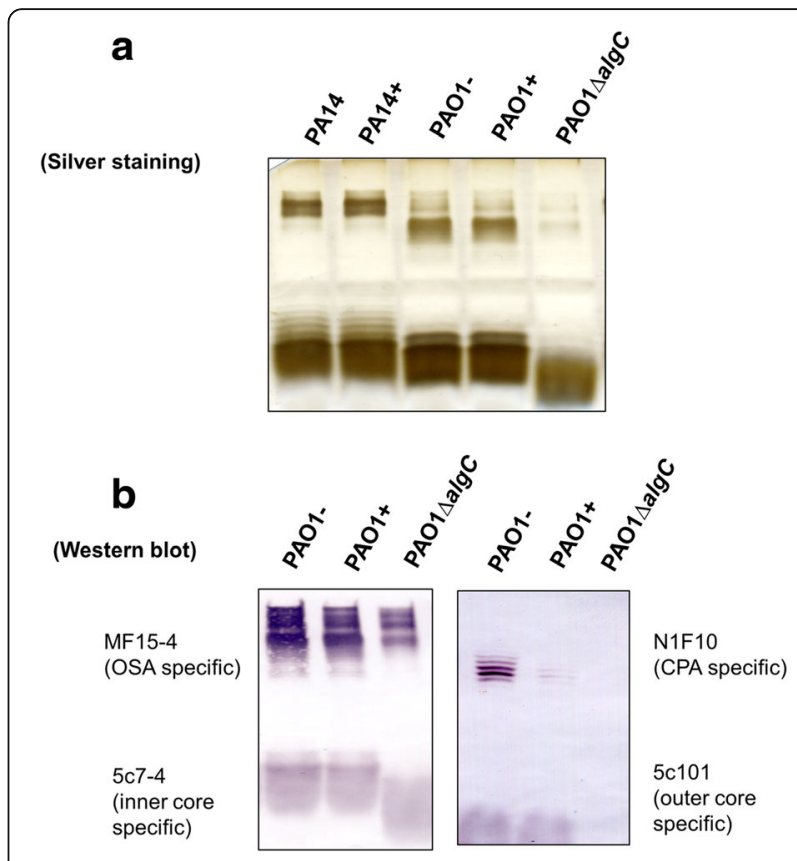

Fig. 5 Analysis of LPS preparations from PAO1 and PA14 strains with (+) or without (-) PAPI-1. a LPS silver staining. b Western blot with a combination of antibodies specifically recognizing A-band (N1F10), B-band (MF15-4), outer core (5c101) and inner core (5c7-4)

expressed and purified a GST-pilV2' fusion protein and performed ELISA to compare the in vitro binding capacity of pilV2' to LPS derived from PAO1+ and PAO1strains. PilV2' is a small pilin protein constituting the type IVb pilus [15] showing significant similarity to adhesins PilVB and PilVA' of plasmid R64, with 40\% identity in amino acid sequence (Additional file 11: Figure S3). C-terminal variable segments of R64 pilV adhesins were previously shown to interact in vitro with LPS of recipient cells [17]. Therefore, we engineered a glutathione transferase (GST) fusion protein carrying at its C-terminus a 97-amino acid C-terminal segment of PilV2, so-called GST-pilV2' fusion. This is analogous to the construct used to analyze the R64 pilin interactions with its receptor. This GST-PilV2' fusion was then tested for binding to various LPS preparations. The binding capacity of GST-PilV2' to LPS from PAO1+ was significantly decreased compare to binding with LPS from PAO1- (Fig. 6). These observations support the hypothesis that the acquisition of PAPI-1 would result in the modification of PAO1's LPS, which in turn might cause the loss of the ability to interact with the pilus from donor strains.

\section{Discussion}

It is well known that horizontal gene transfer plays an important role in driving bacterial evolution and 


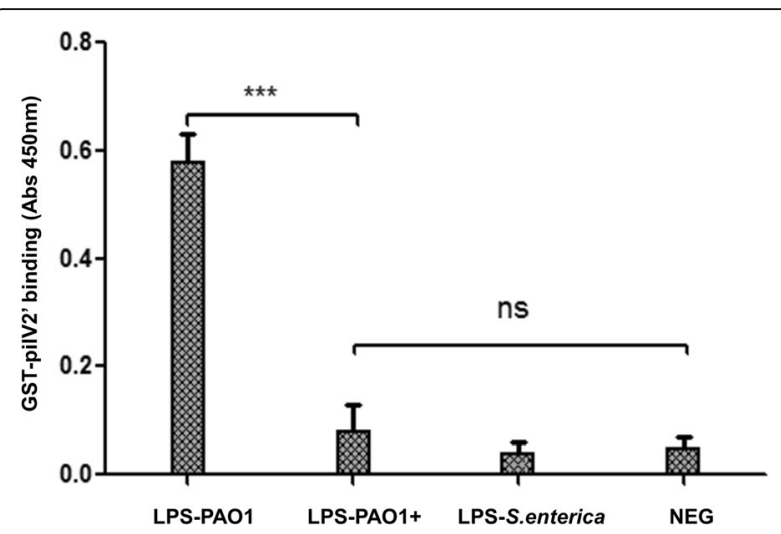

Fig. 6 Binding of GST-pilV2' to various LPS preparations in an enzyme-linked immunosorbent assay. LPS from Salmonella enterica and sample without LPS used as negative control. LPS derived from PAO1 carrying PAPI-1 showed the loss of binding capacity to GST-pilV2'. Results were presented as mean \pm SD for three independent experiments. Statistical significance was assessed by One-way ANOVA (*** $p<0.001$; ns: no significance, $P>0.05$ )

adaptation to survive in various environments. $P$. aeruginosa genome has a mosaic structure composed of a variable number of horizontally-acquired accessory regions containing up to hundreds of genes [12]. PAPI-1 is the largest genomic island in $P$. aeruginosa, and has previously been shown to be transferable to recipients lacking it through direct cell-to-cell interaction and by a conjugation mechanism [15].

Here, we demonstrate, for the first time, that $P$. aeruginosa PAO1 derivatives defective in CPA biosynthesis are significantly affected in their ability to acquire PAPI-1, and that addition of outer membrane or CPA LPS preparations from wild-type PAO1 significantly inhibits PAPI1 transfer. These data suggest that CPA LPS on the recipient membrane plays a significant role during the conjugation and transfer of PAPI-1. LPS has been shown to be the receptor for transfer of different plasmids and bacteriophages. For example, the R64 plasmid transfer is mediated by the recognition of specific parts of the LPS core. Specifically, the GlcNAc $(\alpha 1-2)$ Glc or Glc $(\alpha 1-2) \mathrm{Gal}$ structures, are recognized by PilVB' and PilVC' adhesins, respectively [29]. Moreover, LPS molecules have been found as receptors for many bacteriophages [44]. The D-rhamnose-containing CPA-LPS has been shown to be a receptor for bacteriophage $A 7$, and the phage is capable of hydrolyzing the CPA-LPS to expose core-lipid A [45]. Our data strongly suggests that the conjugative pilus interacts with CPA-LPS on the surface of the recipients in order to initiate the transfer of PAPI-1.

Our work suggests that the PAPI-1 transfer occurs toward another $P$. aeruginosa strain producing CPA LPS or homopolymer of D-rhamnose (D-Rha) structure (from PA14 toward PAO1). CPA is one of the two types of O-polysaccharides (OPS) produced by the majority of $P$. aeruginosa strains [32]. Homologous CPA biosynthesis genes have also been found in the genome of $P$. fluorescens pfO-1 [46], but there is no report about LPS structure of this strain to date. On the other hand, the same structure of D-Rha repeating units $[\rightarrow 3)$ D-Rha $(\alpha 1 \rightarrow 3)$ D$\operatorname{Rha}(\alpha 1 \rightarrow 2)$ D-Rha $(\alpha 1 \rightarrow)]$ has also been found in the LPS of several other bacterial species including the plant pathogen Xanthomonas campestris pv. phaseoli var. fuscans [47], the opportunistic human pathogen Stenotrophomonas maltophilia serotype O7 [48], P. syringae strains [49] and a Burkholderia cepacia strain [50]. The presence of the same LPS structure in different bacterial species raises the possibility that the PAPI-1 island may be transferable among those species. Future studies need to address this intriguing possibility that would enhance our knowledge about the mobilization of genetic elements in bacterial communities in the human body and in the environment.

We also demonstrated that following acquisition of the PAPI-1island, the recipients can become stable donors for transfer of the island to other recipient cells. Remarkably, PAPI-1 acquisition was stable after several generations and in fact during evolution PAPI-1 is partially or entirely retained in some clinical isolates [13]. This would suggest that the acquisition of PAPI-1 would probably be beneficial to the host though it is not well understood in which manner. However, since the size of PAPI-1 is quite large, about $100 \mathrm{~kb}$, it is likely to cause inconvenience to the cells due to metabolic cost and genome expansion. Similarly to conjugative plasmid, the recipients may respond to a number of incoming ICEs [51].

Herein, we demonstrated that the recipient which already acquired PAPI-1 island were able to receive more copies though transfer efficiency was significantly decreased. At present, we have not collected data to define how many copies of PAPI-1 that recipient would have the capacity to receive from the donor strain and how long it might maintain it; however, the occurrence of multiple copies of PAPI-1 at the attB site as a tandem array was previously reported [14]. The pathogenicity islands in P.aeruginosa, PAPI-1 and PAPI-2, are known to be inserted and excised at the specific att sites located in the two tRNA ${ }^{\text {Lys }}$ genes, which were identified as "hot spots" for insertion and excision of large genetic elements in several $P$. aeruginosa strains [14]. For instance, the large plasmid pKLK106 in P. aeruginosa clone K was able to recombine sequentially with either of the two tRNA $^{\text {Lys }}$ genes PA4541.1 and PA0976.1 to rearrange the genomes of sequential $\mathrm{K}$ isolates from the airway of a CF patient [52]. In $P$. aeruginosa clone $C$, the plasmid pKLC102 reversibly integrated only into PA4541.1 but not into PA0976.1, which was occupied by a 23-kb PAGI-4 island [53]. In Vibrio cholerae, it was reported that tandem arrays of SXT and R391 elements occurred 
after their transfer, and this arrangement was observed to be stably maintained for many generations [54]. These suggested that the attB site in a the genome of a recipient can be used as a platform to build composite GIs by sequentially acquired independent genetic elements to form a superintegron [55]. Finally, the integration of PAPI-1 into the site for PAPI-2 means that this attB site is conserved and remains intact at the borders of the composite element. This feature could be used for acquiring multiple ICEs in P. aeruginosa. Harboring at least two "hot spots" for integration of genetic elements, this bacterium is likely to employ an exclusion system to avoid the expansion of its genome and metabolism. This activity has been well documented for conjugative plasmids, but there is not much evidence of this for ICEs. After acquiring a genetic element, the bacteria can modify their cell surfaces or express specific factors to ignore or cleave incoming elements, which are classified in different barrier levels. The bacteria might possess and activate one or some of them to maintain a stable state, and this would affect the efficiency of the transfer after acquiring these elements. In this study, the exclusion activity of PA14 and PAO1 with PAPI-1 were successfully addressed. Exclusion Index (EI) was calculated as the transfer efficiency of PAPI-1 to the recipient lacking this element divided by that to a recipient already carrying the same element [51]. We found that the EI for mating between PAO1+ donor and PAO1+ recipient was about 298. The observed EI values were comparable to those of the SXT family of ICEs [56], but about two-fold lower than that reported for the virulence plasmid pVAPA1037 [57], six-fold lower than for the plasmid R27-mediated entry exclusion [58], and twenty-fold lower than for the highly promiscuous plasmid RP4, which has EI values ranging from $10^{3}$ to $10^{4}$ [59]. Moreover, we found that addition of LPS preparations of PAO1- significantly inhibits transfer efficiency of PAPI-1, while addition of LPS preparations of PAO1+ has no significant effect on transfer efficiency of PAPI-1. When we examined the LPS of PAO1 strains, we found that upon acquisition of PAPI1 , PAO1+ reduces the amount of CPA, and this presumably adversely affects the tightness of the contact between donor and recipient cells. These data indicate that reduction of CPA LPS after acquisition of PAP1-1 serves as an exclusion mechanism for acquisition of multiple copies of the PAPI-1 island. It is worth noting that PA14 strain contains a SNP in the CPA biosynthesis gene $w b p X$ and naturally does not produce CPA [60]. In fact, in accordance with our proposed role of the reduction of CPA as an exclusion mechanism, we found that the EI value for mating between PA14 donor and PA14+ recipient was 12, which is an order of magnitude lower than that measured for PAO1. Unlike the LPS preparation of PAO1-, the LPS from PA14- did not induce any significant variation in transfer efficiency. Interestingly, the outer membrane preparations of PA14- showed some inhibitory effect to PAPI-1 transfer efficiency, while that of PA14+ did not. This may suggest that in addition to CPA, there might be other exclusion mechanisms present in the outer membrane of this strain.

To date, at least two exclusion mechanisms for plasmid or ICEs acquisition are known. One is the entry exclusion (Eex) mediated by inner membrane proteins. This mechanism is able to inhibit DNA entry after a stable mating pair has been established $[56,59,61]$. The other mechanism occurs via surface exclusion, inhibiting formation of a stable mating pair. An example is TraT, which is an outer membrane protein encoded by the $\mathrm{F}$ plasmid. This protein can impede the interaction between the pilus tip and OmpA receptor in E. coli [61-63].

More work is required to elucidate the molecular basis for the PAPI-1 exclusion mechanism. Considering that PAPI-1 lacks any identifiable homologues of genes involved in LPS biosynthesis and modification (data not shown), the factors causing a reduction in the amount of CPA LPS may be conceivably located in the core genome of $P$. aeruginosa. PAPI-1 could therefore specify regulatory functions controlling the expression of the enzymes for LPS biosynthesis. Our study provides new insights on the horizontal acquisition and exclusion of genomic islands, which may lead to future development of new strategies to limit the spread of virulence or resistance functions in $P$. aeruginosa populations.

\section{Conclusions}

Horizontal gene transfer (HGT) represents a major evolutionary mechanism for the acquisition of new phenotypes by microorganisms. HGT plays a particularly important role in the evolution of virulence and antibiotic resistance as it allows acquisition of genes that can alter the pathogenic potential of a bacterial strain. To our knowledge, the mechanism of HGT has never been experimentally investigated in $P$. aeruginosa. This may be partly due to the fact that the mobility of these elements is frequently lost because of evolutionary decay. The significance of this work is in our ability to experimentally test the molecular mechanisms of acquisition of the genomic island PAPI-1 by HGT. Indeed, we provided evidence that conjugative type IVb pilus recognizes CPA lipopolysaccharide of recipient cells to initiate PAPI-1 pathogenicity island transfer in $P$. aeruginosa. We also report that the bacterium produces less CPA after acquiring PAPI-1, as a mechanism to exclude the acquisition of additional copies of PAPI-1. New insights about PAPI-1 mobility and its dissemination by HGT could be applicable to other systems where experimental validation of transmission models has not yet been attained. 


\section{Additional files}

Additional file 1: Table S1. Strains and plasmids used in this study. (DOC $56 \mathrm{~kb}$ )

Additional file 2: Table S4. Primers used in this study. (DOC $45 \mathrm{~kb}$ ) Additional file 3: Table S2. List of PAO1 mutants for lipopolysaccharide biosynthesis. (DOC 67 kb)

Additional file 4: Table S3. Genes involved or potentially involved in Common Polysaccharide Antigen (A-band LPS) biosynthesis. This table was reproduced by King et al., 2009. (DOC 69 kb)

Additional file 5: Figure S1. The GDP-D-rhamnose (CPA monomer) biosynthesis pathway. All the enzymes are encoded by a gene cluster, except the second enzyme phosphomannomutase encoded by algC gene in the alginate locus. D-fructose-6-phosphate; 1, Pi, phosphate; 2, mannose-6-Pi; 3, GDP-D-Man; 4, a-mannose-1-Pi; 5, GDP-4-keto-D-Rha; 6. This figure was modified from King et al., 2009 [32]. (TIF 572 kb)

Additional file 6: Figure S2. Role of psIB gene in PAPI-1 transfer. Results were shown as mean \pm SD for three independent replicates. Statistical significance was analyzed by One-way ANOVA compared to the positive control (ns: no significant difference; and ${ }^{* *} p<0.001$ ). (TIF $217 \mathrm{~kb}$ )

Additional file 7: Table S5. Transfer efficiency of different strains and mutants. (DOCX $19 \mathrm{~kb}$ )

Additional file 8: Table S7. PAPI-1 transfer inhibition following addition of OMs. (DOCX $24 \mathrm{~kb}$ )

Additional file 9:Table S8. PAPI-1 transfer inhibition following addition of LPSs. (DOCX $22 \mathrm{~kb}$ )

Additional file 10: Table S6. Transfer efficiency of different combinations of donors and recipients with or without PAPI-1. (DOCX $14 \mathrm{~kb}$ )

Additional file 11: Figure S3. Homologues of P.aeruginosa pilV2 sequence and pilV adhesins of plasmid R64 of Salmonella enterica serovar Typhimurium LT2 (Multiple Sequence Alignment, Clustal Omega software, EMBL-EBI). (TIF $2355 \mathrm{~kb})$

\section{Acknowledgments}

We are grateful to Alessandra Polissi for helping us with LPS preparations and to Dan Wozniak for kindly providing us with the wbpW/pslB double mutant.

\section{Funding}

This work was supported by AREA1 104038 PhD fellowship assigned to THP from the European Commission, and by OJ's startup fund at the Centre for Integrative Biology. The funders had no role in study design, data collection and interpretation, or on the decision to submit the work for publication. Work in the laboratory of JSL is funded by the Canadian Institutes of Health Research (MOP-14687). YH is a recipient of a Cystic Fibrosis Canada postdoctoral fellowship, and JSL holds a Canada Research Chair in Cystic Fibrosis and Microbial Glycobiology from the Canadian Foundation of Innovation.

\section{Availability of data and materials}

All data generated and analysed during this study are included in this published article.

\section{Authors' contributions}

$\mathrm{TPH}, \mathrm{SL}$ and OJ designed research; TPH performed all experiments with the assistance of SC, PS at the lab of OJ and of MC at the lab of SL, with the exception of the data presented in Fig. 5 which was obtained by $\mathrm{YH}$ and $\mathrm{J}$. $\mathrm{TH}, \mathrm{PS}, \mathrm{SL}, \mathrm{J}$ and OJ wrote the manuscript. All authors have read and approved the final manuscript.

\section{Competing interests}

The authors declare that they have no competing interests.

\section{Consent for publication}

Not applicable.

Ethics approval and consent to participate

Not applicable.

\section{Author details}

${ }^{1}$ Centre for Integrative Biology, University of Trento, 38123 Trento, Italy. Department of Microbiology and Immunobiology, Harvard Medical School, Boston, MA 02115, USA. ${ }^{3}$ Department of Molecular and Cellular Biology, University of Guelph, Guelph, ON N1G 2W1, Canada.

Received: 24 August 2016 Accepted: 3 February 2017

Published online: 07 February 2017

\section{References}

1. Hacker J, Kaper JB. Pathogenicity islands and the evolution of microbes. Annu Rev Microbiol. 2000;54:641-79.

2. Croucher $\mathrm{NJ}$, et al. Role of conjugative elements in the evolution of the multidrug-resistant pandemic clone Streptococcus pneumoniaeSpain23F ST81. J Bacteriol. 2009:191(5):1480-9.

3. Imamovic L, et al. Ol-57, a genomic island of Escherichia coli O157, is present in other seropathotypes of Shiga toxin-producing E. coli associated with severe human disease. Infect Immun. 2010;78(11):4697-704.

4. Gartemann $\mathrm{KH}$, et al. The genome sequence of the tomato-pathogenic actinomycete Clavibacter michiganensis subsp. michiganensis NCPPB382 reveals a large island involved in pathogenicity. J Bacteriol. 2008;190(6): 2138-49.

5. Hacker J, et al. Pathogenicity islands of virulent bacteria: structure, function and impact on microbial evolution. Mol Microbiol. 1997;23(6):1089-97.

6. Dobrindt $U$, et al. Genomic islands in pathogenic and environmental microorganisms. Nat Rev Microbiol. 2004;2(5):414-24.

7. Burrus V, Marrero J, Waldor MK. The current ICE age: biology and evolution of SXT-related integrating conjugative elements. Plasmid. 2006;55(3):173-83.

8. Burrus $\mathrm{V}$, et al. Conjugative transposons: the tip of the iceberg. Mol Microbiol. 2002;46(3):601-10.

9. Kung VL, Ozer EA, Hauser AR. The accessory genome of Pseudomonas aeruginosa. Microbiol Mol Biol Rev. 2010;74(4):621-41.

10. Burrus V, Waldor MK. Shaping bacterial genomes with integrative and conjugative elements. Res Microbiol. 2004;155(5):376-86.

11. Lawley TD, et al. F factor conjugation is a true type IV secretion system. FEMS Microbiol Lett. 2003;224(1):1-15.

12. Mathee $\mathrm{K}$, et al. Dynamics of Pseudomonas aeruginosa genome evolution. Proc Natl Acad Sci U S A. 2008;105(8):3100-5.

13. He J, et al. The broad host range pathogen Pseudomonas aeruginosa strain PA14 carries two pathogenicity islands harboring plant and animal virulence genes. Proc Natl Acad Sci U S A. 2004;101(8):2530-5.

14. Qiu X, Gurkar AU, Lory S. Interstrain transfer of the large pathogenicity island (PAPI-1) of Pseudomonas aeruginosa. Proc Natl Acad Sci U S A. 2006; 103(52):19830-5.

15. Carter MQ, Chen J, Lory S. The Pseudomonas aeruginosa pathogenicity island PAPI-1 is transferred via a novel type IV pilus. J Bacteriol. 2010;192(13): 3249-58.

16. Komano T. Shufflons: multiple inversion systems and integrons. Annu Rev Genet. 1999;33:171-91.

17. Ishiwa A, Komano T. PilV adhesins of plasmid R64 thin pili specifically bind to the lipopolysaccharides of recipient cells. J Mol Biol. 2004;343(3):615-25.

18. Liberati NT, et al. An ordered, nonredundant library of Pseudomonas aeruginosa strain PA14 transposon insertion mutants. Proc Natl Acad Sci U S A. 2006;103(8):2833-8.

19. Hoang TT, et al. A broad-host-range Flp-FRT recombination system for sitespecific excision of chromosomally-located DNA sequences: application for isolation of unmarked Pseudomonas aeruginosa mutants. Gene. 1998;212(1): 77-86.

20. Simon R, Priefer U, Pühler A. A broad host range mobilization system for in vivo genetic engineering: transposon mutagenesis in gram negative bacteria. Bio/Technology. 1983;1(9):784-91.

21. Ravaoarinoro M, et al. Rapid method for isolating detergent-insoluble outer membrane proteins from Pseudomonas aeruginosa. Electrophoresis. 1994; 15(5):594-6.

22. Lam JS, Anderson EM, Hao Y. LPS quantitation procedures. Methods Mol Biol. 2014:1149:375-402.

23. Fomsgaard A, Freudenberg MA, Galanos C. Modification of the silver staining technique to detect lipopolysaccharide in polyacrylamide gels. J Clin Microbiol. 1990;28(12):2627-31.

24. Lee $\mathrm{CH}$, Tsai CM. Quantification of bacterial lipopolysaccharides by the purpald assay: measuring formaldehyde generated from 2-keto-3- 
deoxyoctonate and heptose at the inner core by periodate oxidation. Anal Biochem. 1999;267(1):161-8.

25. Genco CA, Clark VL. Role of outer-membrane proteins and lipopolysaccharide in conjugation between Neisseria gonorrhoeae and Neisseria cinerea. J Gen Microbiol. 1988;134(12):3285-94.

26. Hitchcock PJ, Brown TM. Morphological heterogeneity among Salmonella lipopolysaccharide chemotypes in silver-stained polyacrylamide gels. J Bacteriol. 1983;154(1):269-77.

27. Frangioni JV, Neel BG. Solubilization and purification of enzymatically active glutathione S-transferase (pGEX) fusion proteins. Anal Biochem. 1993; 210(1):179-87.

28. Youn $\mathrm{JH}$, et al. High mobility group box 1 protein binding to lipopolysaccharide facilitates transfer of lipopolysaccharide to CD14 and enhances lipopolysaccharide-mediated TNF-alpha production in human monocytes. J Immunol. 2008;180(7):5067-74.

29. Ishiwa A, Komano T. Thin pilus PilV adhesins of plasmid R64 recognize specific structures of the lipopolysaccharide molecules of recipient cells. J Bacteriol. 2003;185(17):5192-9.

30. Jacobs MA, et al. Comprehensive transposon mutant library of Pseudomonas aeruginosa. Proc Natl Acad Sci U S A. 2003;100(24): 14339-44.

31. Rocchetta HL, Burrows LL, Lam JS. Genetics of O-antigen biosynthesis in Pseudomonas aeruginosa. Microbiol Mol Biol Rev. 1999;63(3):523-53.

32. King JD, et al. Review: Lipopolysaccharide biosynthesis in Pseudomonas aeruginosa. Innate Immun. 2009;15(5):261-312.

33. Winsor $G \mathrm{~L}$, et al. Enhanced annotations and features for comparing thousands of Pseudomonas genomes in the Pseudomonas genome database. Nucleic Acids Res. 2016;44(D1):D646-53.

34. Hao $Y$, et al. Five new genes are important for common polysaccharide antigen biosynthesis in Pseudomonas aeruginosa. MBio. 2013;4(1):e00631-12.

35. Choudhury B, Carlson RW, Goldberg JB. The structure of the lipopolysaccharide from a galU mutant of Pseudomonas aeruginosa serogroup-011. Carbohydr Res. 2005;340(18):2761-72.

36. Yokota S, et al. The structure of the O-specific chain of lipopolysaccharide from Pseudomonas aeruginosa IID 1008 (ATCC 27584). J Biochem. 1986;99(6):1551-61.

37. Rocchetta HL, Pacan JC, Lam JS. Synthesis of the A-band polysaccharide sugar D-rhamnose requires Rmd and WbpW: identification of multiple AlgA homologues, WbpW and ORF488, in Pseudomonas aeruginosa. Mol Microbiol. 1998;29(6):1419-34.

38. King JD, et al. The structural basis for catalytic function of GMD and RMD, two closely related enzymes from the GDP-D-rhamnose biosynthesis pathway. FEBS J. 2009;276(10):2686-700.

39. Byrd MS, et al. Genetic and biochemical analyses of the Pseudomonas aeruginosa Psl exopolysaccharide reveal overlapping roles for polysaccharide synthesis enzymes in Psl and LPS production. Mol Microbiol. 2009;73(4):622-38.

40. Forsberg LS, Carlson RW. The structures of the lipopolysaccharides from Rhizobium etli strains CE358 and CE359. The complete structure of the core region of R. etli lipopolysaccharides. J Biol Chem. 1998;273(5):2747-57.

41. Huszar $\mathrm{G}$, et al. Detection of pyrogens in intravenous lg $\mathrm{G}$ preparations. Biologicals. 2002;30(2):77-83.

42. Ochiai $M$, et al. Interfering effect of diphtheria-tetanus-acellular pertussis combined (DTaP) vaccines on the bacterial endotoxin test. Biologicals. 2001;29(1):55-8.

43. Bang FB. A bacterial disease of Limulus polyphemus. Bull Johns Hopkins Hosp. 1956;98(5):325-51.

44. Picken RN, Beacham IR. Bacteriophage-resistant mutants of Escherichia coli K12. Location of receptors within the lipopolysaccharide. J Gen Microbiol. 1977;102(2):305-18.

45. Rivera M, et al. Common antigen lipopolysaccharide from Pseudomonas aeruginosa AK1401 as a receptor for bacteriophage A7. J Bacteriol. 1992;174(7):2407-11.

46. Silby MW, et al. Genomic and genetic analyses of diversity and plant interactions of Pseudomonas fluorescens. Genome Biol. 2009;10(5):R51.

47. Senchenkova SN, et al. Structures of the O-polysaccharide chains of the lipopolysaccharides of Xanthomonas campestris pv phaseoli var fuscans GSPB 271 and X campestris pv malvacearum GSPB 1386 and GSPB 2388 Carbohydr Res. 2002;337(19):1723-8.

48. Winn AM, Wilkinson SG. The $\mathrm{O} 7$ antigen of Stenotrophomonas maltophilia is a linear D-rhamnan with a trisaccharide repeating unit that is also present in polymers for some Pseudomonas and Burkholderia species. FEMS Microbiol Lett. 1998;166(1):57-61.

49. Ovod V, et al. Immunochemical characterization of $O$ polysaccharides composing the alpha-D-rhamnose backbone of lipopolysaccharide of Pseudomonas syringae and classification of bacteria into serogroups 01 and O2 with monoclonal antibodies. J Bacteriol. 1996;178(22):6459-65.

50. Cerantola S, Montrozier H. Structural elucidation of two polysaccharides present in the lipopolysaccharide of a clinical isolate of Burkholderia cepacia. Eur J Biochem. 1997;246(2):360-6.

51. Garcillan-Barcia MP, de la Cruz F. Why is entry exclusion an essential feature of conjugative plasmids? Plasmid. 2008;60(1):1-18.

52. Kiewitz C, et al. Monitoring genome evolution ex vivo: reversible chromosomal integration of a $106 \mathrm{~kb}$ plasmid at two tRNA(Lys) gene loci in sequential Pseudomonas aeruginosa airway isolates. Microbiology. 2000;146(Pt 10):2365-73.

53. Klockgether J, et al. Sequence analysis of the mobile genome island pKLC102 of Pseudomonas aeruginosa C. J Bacteriol. 2004;186(2):518-34.

54. Hochhut B, et al. Formation of chromosomal tandem arrays of the SXT element and R391, two conjugative chromosomally integrating elements that share an attachment site. J Bacteriol. 2001;183(4):1124-32.

55. Rowe-Magnus DA, Mazel D. Integrons: natural tools for bacterial genome evolution. Curr Opin Microbiol. 2001:4(5):565-9.

56. Marrero J, Waldor MK. Interactions between inner membrane proteins in donor and recipient cells limit conjugal DNA transfer. Dev Cell. 2005;8(6): 963-70.

57. Tripathi VN, et al. Conjugal transfer of a virulence plasmid in the opportunistic intracellular actinomycete Rhodococcus equi. J Bacteriol. 2012;194(24):6790-801.

58. Gunton JE, et al. Entry exclusion in the IncHI1 plasmid R27 is mediated by EexA and EexB. Plasmid. 2008;59(2):86-101.

59. Haase J, Kalkum M, Lanka E. TrbK, a small cytoplasmic membrane lipoprotein, functions in entry exclusion of the IncP alpha plasmid RP4. J Bacteriol. 1996;178(23):6720-9.

60. Hao Y, et al. Single-nucleotide polymorphisms found in the migA and wbpX glycosyltransferase genes account for the intrinsic lipopolysaccharide defects exhibited by pseudomonas aeruginosa PA14. J Bacteriol. 2015; 197(17):2780-91.

61. Sukupolvi S, O'Connor CD. TraT lipoprotein, a plasmid-specified mediator of interactions between gram-negative bacteria and their environment. Microbiol Rev. 1990;54(4):331-41.

62. Achtman M, Kennedy N, Skurray R. Cell-cell interactions in conjugating Escherichia coli: role of traT protein in surface exclusion. Proc Natl Acad Sci U S A. 1977;74(11):5104-8.

63. Riede I, Eschbach ML. Evidence that TraT interacts with OmpA of Escherichia coli. FEBS Lett. 1986;205(2):241-5.

\section{Submit your next manuscript to BioMed Central and we will help you at every step:}

- We accept pre-submission inquiries

- Our selector tool helps you to find the most relevant journal

- We provide round the clock customer support

- Convenient online submission

- Thorough peer review

- Inclusion in PubMed and all major indexing services

- Maximum visibility for your research

Submit your manuscript at www.biomedcentral.com/submit 